\title{
Contrast-Enhanced Harmonic Endoscopic Ultrasound
}

National Cancer Institute

\section{Source}

National Cancer Institute. Contrast-Enhanced Harmonic Endoscopic Ultrasound. NCI

Thesaurus. Code C116502.

A type of doppler ultrasound that uses microbubble contrast to image perfusion and microvasculature within tissues. 